Review article

\title{
Mechanisms of quinolone resistance in Salmonella
}

\author{
Axel CloecKaerT*, Elisabeth Chaslus-Dancla \\ Station de Pathologie Aviaire et Parasitologie, Institut National de la Recherche Agronomique, \\ 37380 Nouzilly, France
}

(Received 20 February 2001; accepted 16 March 2001)

\begin{abstract}
As in other Gram-negative bacteria, mechanisms of resistance to quinolones in Salmonella include target gene mutations, active efflux, and decreased outer membrane permeability. However, the exact contribution of these individual mechanisms to resistance, which may nevertheless interplay to reach high-level resistance, has not yet clearly been defined as in other bacteria such as Escherichia coli. This paper reviews the current state of knowledge of quinolone resistance mechanisms in Salmonella by comparison with that of E. coli and future directions of research with particular attention to the recent development of efflux pump inhibitors as possible means of avoiding the emergence and spread of fluoroquinolone resistance.
\end{abstract}

Salmonella / quinolone / resistance / mechanism

Résumé - Mécanismes de résistance aux quinolones chez Salmonella. Comme chez les autres bactéries Gram-négatives les mécanismes de résistance aux quinolones chez Salmonella comprennent les mutations dans les gènes cibles, l'efflux actif, et une diminution de la perméabilité de la membrane externe. Cependant la contribution exacte de ces mécanismes individuels, qui peuvent néanmoins interagir pour atteindre des niveaux de résistance élevés, n'a pas encore été clairement définie comme chez d'autres bactéries telle que Escherichia coli. Cette revue présente l'état actuel des connaissances des mécanismes de résistance chez Salmonella par comparaison avec E. coli et des perspectives futures de recherche avec une attention particulière au développement récent d'inhibiteurs de pompes d'efflux comme moyen possible pour éviter l'émergence et la diffusion de la résistance aux fluoroquinolones.

Salmonella / quinolone / résistance / mécanisme

*Correspondence and reprints

Tel.: (33) 2474277 50; fax: (33) 2474277 74; e-mail: cloeckae@ @ours.inra.fr 
Table of contents

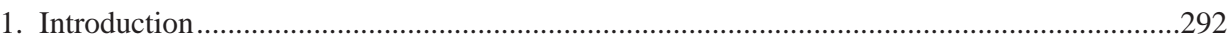

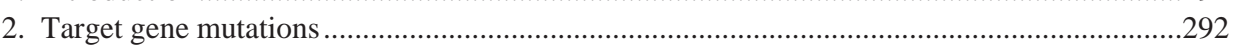

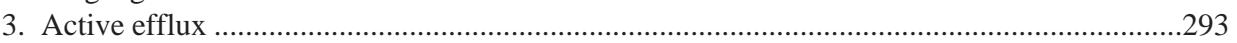

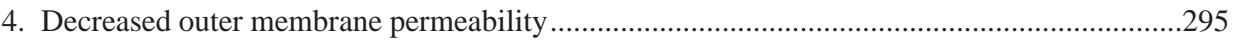

5. How can high-level quinolone resistance be reached?................................................295

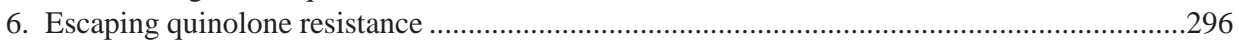

\section{INTRODUCTION}

The evolution of resistance to fluoroquinolones is a problem in both veterinary and human medicine. Over the last years, we have faced the emergence of strains of Escherichia coli highly resistant to fluoroquinolones in humans as well as in different types of food animal production. The situation in Salmonella seems to be different. In humans and animals, a similar emergence of strains showing high-level resistance to fluoroquinolones is not frequently encountered. To date, only one example has been recorded with the emergence and probable clonal diffusion of the $S$. enterica Typhimurium variant Copenhagen in limited areas of Europe. These strains, which are highly resistant to fluoroquinolones, were mainly isolated between 1991 and 1995 from animals and humans [23, 25, 27]. Since then, at least in France, only certain strains with a decreased susceptibility to fluoroquinolones have been isolated [17].

As in other bacteria, mechanisms of resistance to quinolones in Salmonella include target gene mutations, active efflux, and decreased outer membrane permeability. However, the exact contribution of these individual mechanisms to resistance, which may nevertheless interplay to reach high level resistance, has not yet clearly been defined as in other bacteria such as E. coli. The following paragraphs will deal with the current state of knowledge of resistance mechanisms of Salmonella, points of discussion and future directions of research by comparison with the better studied individ- ual quinolone resistance mechanisms in other bacteria such as E. coli.

\section{TARGET GENE MUTATIONS}

In Salmonella, quinolone resistance was firstly attributed to point mutations in the gyrA gene coding for the A subunit of gyrase, whose complex with DNA is the primary target of quinolones. Resistance mutations of gyrA have been clustered in a region of the gene product between amino acids 67 and 106, termed the quinolone resistance-determining region (QRDR). Amino acid changes at Ser-83 (to Phe, Tyr, or Ala) or at Asp-87 (to Gly, Asn, or Tyr) are the most frequently observed in nalidixic acid-resistant strains $[6,17,20,21,26,54$, 57, 63-65, 70, 72]. Double mutations at both residues 83 and 87 have been identified in clinical isolates of $S$. enterica serovar Typhimurium showing high-level resistance to fluoroquinolones (e.g., MIC of ciprofloxacin: $32 \mu \mathrm{g} \cdot \mathrm{mL}^{-1}$ ) [23]. This concerned a particular clone named Copenhagen variant encountered in Germany during the early 1990s. The double mutation was identical in several isolates (Ser-83 to Ala and Asp-87 to Asn) thereby suggesting the clonality of the strains [25]. These isolates additionally had an altered $g y r B$ gene coding for the B subunit of gyrase [23]. Complementation of these strains with plasmids carrying either the $g y r A$ or the $g y r B$ wild-type genes of $E$. coli $\mathrm{K}-12$ resulted in enhanced susceptibilities and provided evidence of a role for both altered gyrA and $g y r B$ genes in 
high-level fluoroquinolone resistance [23]. Probably the same clone described as phage-type DT204 showing high-level enrofloxacin resistance occurred in Belgium from 1991 to 1995 [27]. Mutations in the target genes were, however, not investigated in these cases. This clone has apparently disappeared and only clinical strains with a single mutation in the gyrA gene have since then been described to confer nalidixic acid resistance but decreased susceptibility to fluoroquinolones according to the clinical breakpoint admitted (MIC $>2 \mu \mathrm{g} \cdot \mathrm{mL}^{-1}$ ). A gyrB gene mutation has also been identified in a quinolone-resistant post-therapy isolate of S. enterica serovar Typhimurium and consisted in a point mutation in codon 463 of $\operatorname{gyr} B$ giving rise to an amino acid substitution of Ser to Tyr [16]. In this case, complementing with a wild-type gyrB gene also resulted in increased susceptibility of the isolate to nalidixic acid and fluoroquinolones.

Less frequently, amino acid substitutions at other GyrA positions than Ser-83 and Asp-87 have been described in quinoloneresistant Salmonella strains such as those at Ala-67 (to Pro), Gly-81 (to Ser), and Ala119 (to Glu) [17, 21, 63].

Unlike in E. coli [12, 14, 19, 24, 28, 32, $44,51]$ no mutations have been described in the secondary target for quinolones, namely the parC gene coding for the ParC subunit of topoisomerase IV.

The role in Salmonella quinolone resistance of mutations in the target genes, in particular in $g y r A$ and $g y r B$, has been confirmed by complementation studies using wild-type genes on plasmids [16, 20, 23, $54,56,57]$. It nevertheless remains to be determined what the exact impact of these mutations is on quinolone resistance, as has been done for $E$. coli constructed isogenic mutants where mutations in gyrA and parC were experimentally introduced in the absence of quinolone selection [4]. Indeed, nalidixic acid and fluoroquinolones may select for mutations at other chromosomal loci, in particular those resulting in a multiple antibiotic resistance (Mar) phenotype due to the overproduction of broad-spectrum efflux pumps and decreased outer membrane permeability which may interplay with target gene mutation-mediated quinolone resistance (see below) $[1,2,60]$.

\section{ACTIVE EFFLUX}

Quinolone resistance due to active efflux has been well studied in E. coli. A major efflux system involved in multidrug-comprising quinolone-resistance described in $E$. coli is the AcrAB-TolC system which is a three-component structure that crosses both the inner and the outer membrane. AcrB is the inner membrane component which acts as a proton-motive-force-dependent transporter and belongs to the class of resistancenodulation-division (RND) efflux pumps $[49,50,55,60,61,76]$. Its proposed structure consists of 12 transmembrane segments (TMS) or $\alpha$-helices with two large hydrophilic extracytoplasmic domains between TMS 1 and 2 and TMS 7 and 8 . These large periplasmic domains may play a critical role in the drug efflux. The AcrB transporter uses a wide variety of substrates extruding not only various classes of clinically important antibiotics such as quinolones, $\beta$-lactams, tetracycline, chloramphenicol, erythromycin, and rifampin but also disinfectants, dyes, detergents, bile salts, and organic solvents [29, 40, 43, 48-50, 53, 55, 60, 61, 68, 69, 71].

AcrA is a periplasmic lipoprotein which has an elongated shape thought to span the periplasmic space thus coordinating the concerted operation of inner and outer membrane components of the three-component structure [73, 74]. AcrA, in addition, forms oligomers, most probably trimers interacting specifically with the AcrB transporter independently of substrate and TolC [75]. AcrA might bring TolC and AcrB together by folding back on itself allowing the drug 
efflux across the two membranes directly into the medium [73-76].

The functional outer membrane protein TolC is required for the operation of the AcrAB efflux system and for the expression of the Mar phenotype [15]. The crystal structure of TolC has recently been elucidated revealing a distinctive and previously unknown fold [30]. Three TolC protomers assemble to form a continuous, solvent accessible conduit - a "channel-tunnel" over $140 \AA$ long that spans both the outer membrane and periplasmic space. Trimerisation results in an $\alpha$-helical barrel, which forms a tunnel through the periplasm, anchored by a contiguous outer membrane $\beta$-barrel. The periplasmic end of the tunnel is sealed by sets of coiled helices and it is thought that these could be untwisted by an allosteric mechanism, mediated by protein-protein interactions, to open the tunnel.

In $E$. coli the level of production of the AcrAB-TolC efflux system is controlled by several regulatory genes at different chromosomal loci. The most important systems studied are the global marRAB and soxRS regulatory systems. marA and soxS code for homologous proteins that are positive regulators of the AcrAB-TolC efflux system $[1,2,5,40,41,46,48,53,71]$. The $\operatorname{marR}$ and sox $R$ genes code for repressor proteins that locally downregulate the expression of the marA and soxS genes, respectively. Thus alteration of these repressor genes may lead to constitutive expression of marA and soxS resulting in the overproduction of the AcrAB-TolC efflux pump and resulting Mar phenotype. Upon selection with quinolones, several mutations have been identified in the repressor genes and also in the marO operator upstream of the marRAB operon. These mutations consisted of point mutations and deletions $[1,3,10,28,37,42,51]$. Such non-target gene mutations may thus lead to increased efflux-mediated quinolone resistance. Interestingly, MarA and SoxS also downregulate the synthesis of the major porin OmpF, through the increased production of an antisense RNA, micF, which results in decreased outer membrane permeability to quinolones and other antibiotics $[8,9]$. Besides overproduction of the AcrAB-TolC efflux pump, it has recently been shown using macroarrays that $E$. coli strains constitutively expressing marA showed altered expression of more than 60 chromosomal genes [5].

Closely linked to the $a c r A B$ operon, is the $a c r R$ gene coding for a repressor of acrAB. Apart from the marRAB and soxRS regulons, AcrR would function as a specific secondary modulator to finely tune the level of $a c r A B$ transcription and to prevent the unwanted overexpression of $a c r A B$ [41].

In Salmonella, evidence for the participation of active efflux in quinolone resistance has recently been provided $[18,58]$. Although no direct evidence has been provided for the role of the AcrAB-TolC efflux system in resistance, the production level of the AcrA protein in in vitro quinoloneselected mutants correlated well with the resistance levels to nalidixic acid and fluoroquinolones [18]. As in E. coli, quinoloneselection also resulted in a Mar phenotype with resistance to unrelated antibiotics such as $\beta$-lactams, chloramphenicol, and tetracycline. Interestingly, the Salmonella acrB gene has been shown to play an important role in resistance to bile salts, detergents and in mice intestinal colonisation [34]. A $S$. enterica serovar Typhimurium $a c r B$ mutant indeed exhibited a reduced capacity to colonise the intestinal tract. Similarly, the TolC protein of $S$. enterica serovar Enteritidis has been shown to be required for virulence in mice [66]. The tolC mutant was avirulent for mice when administered by the oral route. This mutant was also more sensitive than the wild-type strain to various detergents, antibiotics, and dyes suggesting that as in E. coli the TolC outer membrane channel of Salmonella is required for effective functioning of the AcrAB-TolC efflux system. These data suggest an important role of the AcrAB-TolC efflux system in the virulence of Salmonella. 
The marRAB and soxRS operons also occur in Salmonella [11, 31, 33, 58, 59, 67]. As in E. coli, increased expression of marA and soxS results in the Mar phenotype [11, 31, 59]. Recently, Koutsolioutsou et al. [31] reported a soxRS-constitutive mutation in a clinical strain of $S$. enterica serovar Typhimurium that arose with the development of resistance to quinolones during treatment. The elevated quinolone resistance in this strain derived from a point mutation in the sox $R$ gene and could be suppressed in trans by multicopy wild-type soxRS thus showing that constitutive expression of soxRS can contribute to antibiotic resistance in clinically relevant $S$. enterica [31]. In another recent study of Piddock et al. [58], evidence has been reported of a role of active efflux in the resistance phenotype of six isolates of $S$. enterica serovar Typhimurium recovered from a patient treated with ciprofloxacin. However, no mutations were found in the marO, marR, and $a c r R$ sequences and no increased expression of marA, tolC, or soxS was observed by Northern blotting although three of the six isolates showed increased expression of $a \operatorname{cr} B$ [58]. To our knowledge these are the only two reports where mutations in the regulator genes of the AcrAB-TolC efflux system have been investigated. Thus much work remains to be done to study the role of efflux and its regulation in Salmonella quinolone resistance to reach the current state of knowledge of efflux in $E$. coli.

\section{DECREASED OUTER MEMBRANE PERMEABILITY}

A few studies have reported on alterations of outer membrane protein expression or of lipopolysaccharide in quinolone-resistant Salmonella [18, 20, 56, 57]. However, it does not appear clearly from these studies whether such alterations contributed significantly to decreased outer membrane permeability and consecutive quinolone resis- tance. The role of lipopolysaccharide composition on the accumulation of quinolones has been studied in several bacteria, but it remains unclear, as contradictory results have been obtained [7, 13, 22, 45, 47, 62]. It has been hypothesised in quinolone-selected Pseudomonas aeruginosa strains that increased amounts of lipopolysaccharide form a permeability barrier which acts preferentially against hydrophilic quinolones [45]. The lengthening of the $\mathrm{O}$ chains seen in in vitro quinolone-selected resistant Salmonella mutants could also result in a lower level of accessibility to the outer membrane [18].

It is thought that hydrophilic quinolones like ciprofloxacin preferentially use the porin pathway to penetrate the cells [7]. Lack of expression of the OmpF porin has been reported in some quinolone-resistant Salmonella strains [20, 56, 57]. However, it was not clear whether the lack of OmpF contributed to decreased levels of quinolone accumulation. As described for E. coli above, it has been shown for Salmonella that SoxS upregulates micF transcription [59] which may be responsible for the lack of expression of OmpF for these quinoloneresistant Salmonella isolates.

\section{HOW CAN HIGH-LEVEL QUINOLONE RESISTANCE BE REACHED?}

Interplay between several resistance mechanisms may lead to high-level resistance to quinolones and to other antibiotics when multidrug efflux pumps and decreased outer membrane permeability are involved $[14,28,35,36,46]$. For instance for in vitro selected quinolone-resistant $E$. coli mutants it has been shown that first-step quinoloneresistant mutants acquire a gyrA mutation [28]. Second-step mutants reproducibly acquire a Mar phenotype and show enhanced fluoroquinolone efflux. In some third-step mutants, fluoroquinolone efflux is further enhanced and additional 
topoisomerase mutations are acquired. In clinical $E$. coli isolates from humans and animals the situation appears to be the same where high-level fluoroquinolone resistance is reached when mutations at several chromosomal loci are acquired [14, 51].

For S. enterica Typhimurium in vitro selected quinolone-resistant mutants, individual mechanisms of resistance also appear step by step and their accumulation also results in high-level fluoroquinolone resistance [18]. However, in contrast to E. coli, active efflux correlating with increased production of the AcrAB efflux pump appears prior to a mutation in gyrA [18]. In addition, no mutation in the parC topoisomerase IV gene occurs in late-step mutants but rather a gradual increase in the production of the AcrAB efflux pump. This suggests a probable step-by-step accumulation of mutations in regulator genes of the AcrAB-TolC efflux system of Salmonella.

High-level fluoroquinolone resistance in clinical isolates of Salmonella has only been reported for the Copenhagen variant clone of S. enterica serovar Typhimurium which appeared during the early 1990s in Germany $[23,25]$ and probably also in Belgium [27]. As described above only mutations in the gyrA and gyrB target genes have been investigated $[23,25]$. It would therefore be worth further studying these isolates to determine whether accumulation of individual resistance mechanisms, in particular active efflux and target gene mutation, resulted in this high-level resistance phenotype.

Over the past few years no high-level fluoroquinolone-resistant clinical strains of Salmonella have been isolated which suggested a counterselection of highly fluoroquinolone-resistant strains in the field [17]. However, this situation might be temporary as the number of nalidixic acid-resistant isolates increases, in particular those of S. enterica serovar Typhimurium phagetype DT104 [70] and particular clones may always emerge showing high-level fluoro- quinolone resistance as was the case in the early 1990s.

\section{ESCAPING QUINOLONE RESISTANCE}

It is particularly interesting to note that in E. coli, inactivation of the AcrAB efflux pump makes resistant strains, including those with target gene mutations, hypersusceptible to fluoroquinolones and certain other unrelated drugs [52]. Thus in the absence of the AcrAB efflux pump, gyrase mutations fail to produce clinically relevant levels of fluoroquinolone resistance [52]. The same observation has been made for Pseudomonas aeruginosa where deletion of the MexAB-OprM efflux pump, which is the homolog of the AcrAB-TolC efflux pump in this species, resulted in a significant decrease in resistance to fluoroquinolones even for strains carrying target gene mutations [38].

Therefore, multidrug efflux pumps such as AcrAB-TolC and MexAB-OprM appear as suitable targets for new therapeutics which, combined with fluoroquinolones, would decrease the level of intrinsic resistance, reverse acquired resistance, and result in a decreased frequency of emergence of fluoroquinolone-resistant strains [38].

One effective inhibitor of multidrug resistance efflux pumps has recently been discovered [39]. This molecule, termed MC207,110, is active against all known Mex efflux pumps from Pseudomonas aeruginosa and the AcrAB-TolC efflux pump homolog of E. coli $[39,44]$. The study of Lomovskaya et al. [39] showed that inhibition of efflux pumps by this molecule indeed (i) significantly decreased the level of intrinsic resistance, (ii) reversed acquired resistance, and (iii) resulted in a decreased frequency of emergence of Pseudomonas aeruginosa strains that are highly resistant to fluoroquinolones. 
Thus the development and application of efflux pump inhibitors as new therapeutics combined with fluoroquinolones appear to be an attractive solution to avoid the emergence of pathogens highly resistant to fluoroquinolones, possibly including Salmonella which should be further investigated.

\section{REFERENCES}

[1] Alekshun M.N., Levy S.B., Regulation of chromosomally mediated multiple antibiotic resistance: the mar regulon, Antimicrob. Agents Chemother. 41 (1997) 2067-2075.

[2] Alekshun M.N., Levy S.B., The mar regulon: multiple resistance to antibiotics and other toxic chemicals, Trends Microbiol. 7 (1999) 410-413.

[3] Ariza R.R., Cohen S.P., Bachhawat N., Levy S.B., Demple B., Repressor mutations in the $\operatorname{mar} R A B$ operon that activate oxidative stress genes and multiple antibiotic resistance in Escherichia coli, J. Bacteriol. 176 (1994) 143-148.

[4] Bagel S., Hullen V., Wiedemann B., Heisig P., Impact of gyrA and parC mutations on quinolone resistance, doubling time, and supercoiling degree of Escherichia coli, Antimicrob. Agents Chemother. 43 (1999) 868-875.

[5] Barbosa T.M., Levy S.B., Differential expression of over 60 chromosomal genes in Escherichia coli by constitutive expression of MarA, J. Bacteriol. 182 (2000) 3467-3474.

[6] Brown J.C., Thomson C.J., Amyes S.G.B., Mutations of the gyrA gene of clinical isolates of Salmonella typhimurium and three other Salmonella species leading to decreased susceptibilities to 4-quinolone drugs, J. Antimicrob. Chemother. 37 (1996) 351-356.

[7] Chapman J.S., Georgopapadakou N.H., Routes of quinolone permeation in Escherichia coli, Antimicrob. Agents Chemother. 32 (1988) 438442.

[8] Cohen S.P., McMurry L.M., Levy S.B., marA locus causes decreased expression of OmpF porin in multiple-antibiotic-resistant (Mar) mutants of Escherichia coli, J. Bacteriol. 170 (1988) 54165422.

[9] Cohen S.P., McMurry L.M., Hooper D.C., Wolfson J.S., Levy S.B., Cross-resistance to fluoroquinolones in multiple-antibiotic-resistant (Mar) Escherichia coli selected by tetracycline or chloramphenicol: decreased drug accumulation associated with membrane changes in addition to $\mathrm{OmpF}$ reduction, Antimicrob. Agents Chemother. 33 (1989) 1318-1325.

[10] Cohen S.P., Hachler H., Levy S.B., Genetic and functional analysis of the multiple antibiotic resis- tance (mar) locus in Escherichia coli, J. Bacteriol. 175 (1993) 1484-1492.

[11] Cohen S.P., Yan W., Levy S.B., A multidrug resistance regulatory chromosomal locus is widespread among enteric bacteria, J. Infect. Dis. 168 (1993) 484-488.

[12] del Mar Tavio M., Vila J., Ruiz J., MartinSanchez A.M., Jimenez de Anta M.T., Mechanisms involved in the development of resistance to fluoroquinolones in Escherichia coli isolates, J. Antimicrob. Chemother. 44 (1999) 735-742.

[13] Denis A., Moreau N.J., Mechanisms of quinolone resistance in clinical isolates: accumulation of sparfloxacin and of fluoroquinolones of various hydrophobicity, and analysis of membrane composition, J. Antimicrob. Chemother. 32 (1993) 379-392.

[14] Everett M.J., Jin Y.F., Ricci V., Piddock L.J.V., Contributions of individual mechanisms to fluoroquinolone resistance in 36 Escherichia coli strains isolated from humans and animals, Antimicrob. Agents Chemother. 40 (1996) 2380-2386.

[15] Fralick J.A., Evidence that TolC is required for functioning of the Mar/AcrAB efflux pump of Escherichia coli, J. Bacteriol. 178 (1996) 58035805.

[16] Gensberg K., Jin Y.F., Piddock L.J.V., A novel gyrB mutation in a fluoroquinolone-resistant clinical isolate of Salmonella typhimurium, FEMS Microbiol. Lett. 132 (1995) 57-60.

[17] Giraud E., Brisabois A., Martel J.L., ChaslusDancla E., Comparative studies of mutations in animal isolates and experimental in vitro- and in vivo-selected mutants of Salmonella spp. suggest a counterselection of highly fluoroquinoloneresistant strains in the field, Antimicrob. Agents Chemother. 43 (1999) 2131-2137.

[18] Giraud E., Cloeckaert A., Kerboeuf D., ChaslusDancla E., Evidence for active efflux as the primary mechanism of resistance to ciprofloxacin in Salmonella enterica serovar Typhimurium, Antimicrob. Agents Chemother. 44 (2000) 12231228 .

[19] Giraud E., Leroy-Sétrin S., Flaujac G., Cloeckaert A., Dho-Moulin M., Chaslus-Dancla E., Characterization of high-level fluoroquinolone resistance in Escherichia coli O78:K80 isolated from turkeys, J. Antimicrob. Chemother. 47 (2001) 341-343.

[20] Griggs D.J., Hall M.C., Jin Y.F., Piddock L.J.V., Quinolone resistance in veterinary isolates of salmonella, J. Antimicrob. Chemother. 33 (1994) 1173-1189.

[21] Griggs D.J., Gensberg K., Piddock L.J.V., Mutations in gyrA gene of quinolone-resistant Salmonella serotypes isolated from humans and animals, Antimicrob. Agents Chemother. 40 (1996) 1009-1013. 
[22] Hancock R.E., The bacterial outer membrane as a drug barrier, Trends Microbiol. 5 (1997) 3742 .

[23] Heisig P., High-level fluoroquinolone resistance in a Salmonella typhimurium isolate due to alterations in both gyrA and $g y r B$ genes, J. Antimicrob. Chemother. 32 (1993) 367-377.

[24] Heisig P., Genetic evidence for a role of parC mutations in development of high-level fluoroquinolone resistance in Escherichia coli, Antimicrob. Agents Chemother. 40 (1996) 879-885.

[25] Heisig P., Kratz B., Halle E., Graser Y., Altwegg M., Rabsch W., Faber J.P., Identification of DNA gyrase A mutations in ciprofloxacin-resistant isolates of Salmonella typhimurium from men and cattle in Germany, Microb. Drug Resist. 1 (1995) 211-218.

[26] Heurtin-Le Corre C., Donnio P.Y., Perrin M. Travert M.F., Avril J.L., Increasing incidence and comparison of nalidixic acid-resistant Salmonella enterica subsp. enterica serotype Typhimurium isolates from humans and animals, J. Clin. Microbiol. 37 (1999) 266-269

[27] Imberechts H., D’hooghe I., Bouchet H., Godard C., Pohl P., Apparent loss of enrofloxacin resistance in bovine Salmonella typhimurium strains isolated in Belgium, 1991 to 1998, Vet. Rec. 147 (2000) 76-77.

[28] Kern W.V., Oethinger M., Jellen-Ritter A.S., Levy S.B., Non-target gene mutations in the development of fluoroquinolone resistance in Escherichia coli, Antimicrob. Agents Chemother. 44 (2000) 814-820.

[29] Kopytek S.J., Dyer J.C.D., Knapp G.S., Hu J.C., Resistance to methotrexate due to AcrAB-dependent export from Escherichia coli, Antimicrob. Agents Chemother. 44 (2000) 3210-3212.

[30] Koronakis V., Sharff A., Koronakis E., Luisi B., Hughes C., Crystal structure of the bacterial membrane protein TolC central to multidrug efflux and protein export, Nature 405 (2000) 914-919.

[31] Koutsolioutsou A., Martins E.A., White D.G., Levy S.B., Demple B., A soxRS-constitutive mutation contributing to antibiotic resistance in a clinical isolate of Salmonella enterica (serovar Typhimurium), Antimicrob. Agents Chemother. 45 (2001) 38-43.

[32] Kumagai Y., Kato J., Hoshino K., Akasaka T., Sato K., Ikeda H., Mutants of Escherichia coli DNA topoisomerase IV parC gene, Antimicrob. Agents Chemother. 40 (1996) 710-714.

[33] Kunonga N.I., Sobieski R.J., Crupper S.S., Prevalence of the multiple antibiotic resistance operon $(\operatorname{marRAB})$ in the genus Salmonella, FEMS Microbiol. Lett. 187 (2000) 155-160.

[34] Lacroix F.J., Cloeckaert A., Grépinet O., Pinault C., Popoff M.Y., Waxin H., Pardon P., Salmonella typhimurium acrB-like gene: identification and role in resistance to biliary salts and detergents and in murine infection, FEMS Microbiol. Lett. 135 (1996) 161-167.

[35] Lee A., Mao W., Warren M.S., Mistry A., Hoshino K., Okumura R., Ishida H., Lomovskaya O., Interplay between efflux pumps may provide either additive or multiplicative effects on drug resistance, J. Bacteriol. 182 (2000) 3142-3150.

[36] Li X.Z., Zhang L., Poole K., Interplay between the MexA-MexB-OprM multidrug efflux system and the outer membrane barrier in the multiple antibiotic resistance of Pseudomonas aeruginosa, J. Antimicrob. Chemother. 45 (2000) 433-436.

[37] Linde H.J., Notka F., Metz M., Kochanowsky B., Heisig P., Lehn N., In vivo increase in resistance to ciprofloxacin in Escherichia coli associated with deletion of the C-terminal part of MarR, Antimicrob. Agents Chemother. 44 (2000) 18651868.

[38] Lomovskaya O., Lee A., Hoshino K., Ishida H., Mistry A., Warren M.S., Boyer E., Chamberland S., Lee V.J., Use of a genetic approach to evaluate the consequences of inhibition of efflux pumps in Pseudomonas aeruginosa, Antimicrob. Agents Chemother. 43 (1999) 1340-1346.

[39] Lomovskaya O., Warren M.S., Lee A., Galazzo J., Fronko R., Lee M., Blais J., Cho D., Chamberland S., Renau T., Leger R., Hecker S., Watkins W., Hoshino K., Ishida H., Lee V.J., Identification and characterization of inhibitors of multidrug resistance efflux pumps in Pseudomonas aeruginosa: novel agents for combination therapy, Antimicrob. Agents Chemother. 45 (2001) 105116.

[40] Ma D., Cook D.N., Alberti M., Pon N.G., Nikaido H., Hearst J.E., Genes $a c r A$ and $a c r B$ encode a stress-induced efflux system of Escherichia coli, Mol. Microbiol. 16 (1995) 45-55.

[41] Ma D., Alberti M., Lynch C., Nikaido H., Hearst J.E., The local repressor AcrR plays a modulating role in the regulation of $a c r A B$ genes of Escherichia coli by global stress signals, Mol. Microbiol. 19 (1996) 101-112.

[42] Maneewannakul K., Levy S.B., Identification of mar mutants among quinolone-resistant clinical isolates of Escherichia coli, Antimicrob. Agents Chemother. 40 (1996) 1695-1698.

[43] Mazzariol A., Cornaglia G., Nikaido H., Contributions of the AmpC $\beta$-lactamase and the AcrAB multidrug efflux system in intrinsic resistance of Escherichia coli $\mathrm{K}-12$ to $\beta$-lactams, Antimicrob. Agents Chemother. 44 (2000) 1387-1390.

[44] Mazzariol A., Tokue Y., Kanegawa T.M., Cornaglia G., Nikaido H., High-level fluoroquinolone-resistant clinical isolates of Escherichia coli overproduce multidrug efflux protein AcrA, Antimicrob. Agents Chemother. 44 (2000) 34413443.

[45] Michea-Hamzehpour M., Furet Y.X., Pechere J.C., Role of protein D2 and lipopolysaccharide in diffusion of quinolones through the outer mem- 
brane of Pseudomonas aeruginosa, Antimicrob. Agents Chemother. 35 (1991) 2091-2097.

[46] Miller P.F., Sulavik M.C., Overlaps and parallels in the regulation of intrinsic multiple-antibiotic resistance in Escherichia coli, Mol. Microbiol. 21 (1996) 441-448.

[47] Mitsuyama J., Itoh Y., Takahata M., Okamoto S., Yasuda T., In vitro antibacterial activities of tosufloxacin against and uptake of tosufloxacin by outer membrane mutants of Escherichia coli, Proteus mirabilis, and Salmonella typhimurium, Antimicrob. Agents Chemother. 36 (1992) 20302036.

[48] Moken M.C., McMurry L.M., Levy S.B., Selection of multiple-antibiotic-resistant (Mar) mutants of Escherichia coli by using the disinfectant pine oil: roles of the mar and $a c r A B$ loci, Antimicrob. Agents Chemother. 41 (1997) 2770-2772.

[49] Nikaido H., Multidrug efflux pumps of Gramnegative bacteria, J. Bacteriol. 178 (1996) 58535859 .

[50] Nikaido H., Antibiotic resistance caused by Gramnegative multidrug efflux pumps, Clin. Infect. Dis. 27 Suppl. 1 (1998) S32-S41.

[51] Oethinger M., Podglajen I., Kern W.V., Levy S.B., Overexpression of the marA or soxS regulatory gene in clinical topoisomerase mutants of Escherichia coli, Antimicrob. Agents Chemother. 42 (1998) 2089-2094.

[52] Oethinger M., Kern W.V., Jellen-Ritter A.S., McMurry L.M., Levy S.B., Ineffectiveness of topoisomerase mutations in mediating clinically significant fluoroquinolone resistance in Escherichia coli in the absence of the AcrAB efflux pump, Antimicrob. Agents Chemother. 44 (2000) 10-13.

[53] Okusu H., Nikaido H., AcrAB efflux pump plays a major role in the antibiotic resistance phenotype of Escherichia coli multiple-antibiotic-resistance (Mar) mutants, J. Bacteriol. 178 (1996) 306-308.

[54] Ouabdesselam S., Tankovic J., Soussy C.J., Quinolone resistance mutations in the gyrA gene of clinical isolates of Salmonella, Microb. Drug Resist. 2 (1996) 299-302.

[55] Paulsen I.T., Brown M.H., Skurray R.A., Protondependent multidrug efflux systems, Microbiol. Rev. 60 (1996) 575-608.

[56] Piddock L.J.V., Griggs D.J., Hall M.C., Jin Y.F., Ciprofloxacin resistance in clinical isolates of Salmonella typhimurium obtained from two patients, Antimicrob. Agents Chemother. 37 (1993) 662-666.

[57] Piddock L.J.V., Ricci V., McLaren I., Griggs D.J., Role of mutation in the gyrA and parC genes of nalidixic-acid-resistant salmonella serotypes isolated from animals in the United Kingdom, J. Antimicrob. Chemother. 41 (1998) 635-641.

[58] Piddock L.J.V., White D.G., Gensberg K., Pumbwe L., Griggs D.J., Evidence for an efflux pump mediating multiple antibiotic resistance in Salmonella enterica serovar Typhimurium, Antimicrob. Agents Chemother. 44 (2000) 31183121.

[59] Pomposiello P.J., Demple B., Identification of SoxS-regulated genes in Salmonella enterica serovar Typhimurium, J. Bacteriol. 182 (2000) 23-29.

[60] Poole K., Efflux-mediated resistance to fluoroquinolones in Gram-negative bacteria, Antimicrob. Agents Chemother. 44 (2000) 2233-2241.

[61] Putman M., van Veen H.W., Konings W.N., Molecular properties of bacterial multidrug transporters, Microbiol. Mol. Biol. Rev. 64 (2000) 672-693.

[62] Rajyaguru J.M., Muszynski M.J., Association of resistance to trimethoprim/sulphamethoxazole, chloramphenicol and quinolones with changes in major outer membrane proteins and lipopolysaccharide in Burkholderia cepacia, J. Antimicrob. Chemother. 40 (1997) 803-809.

[63] Reyna F., Huesca M., Gonzalez V., Fuchs L.Y., Salmonella typhimurium gyrA mutations associated with fluoroquinolone resistance, Antimicrob. Agents Chemother. 39 (1995) 1621-1623.

[64] Ridley A., Threlfall E.J., Molecular epidemiology of antibiotic resistance genes in multiresistant epidemic Salmonella typhimurium DT 104, Microb. Drug Resist. 4 (1998) 113-118.

[65] Ruiz J., Castro D., Goni P., Santamaria J.A., Borrego J.J., Vila J., Analysis of the mechanism of quinolone resistance in nalidixic acid-resistant clinical isolates of Salmonella serotype Typhimurium, J. Med. Microbiol. 46 (1997) 623628.

[66] Stone B.J., Miller V.L., Salmonella enteritidis has a homologue of tolC that is required for virulence in BALB/c mice, Mol. Microbiol. 17 (1995) 701-712.

[67] Sulavik M.C., Dazer M., Miller P.F., The Salmonella typhimurium mar locus: molecular and genetic analyses and assessment of its role in virulence, J. Bacteriol. 179 (1997) 1857-1866.

[68] Thanassi D.G., Cheng L.W., Nikaido H., Active efflux of bile salts by Escherichia coli, J. Bacteriol. 179 (1997) 2512-2518.

[69] Tsukagoshi N., Aono R., Entry into and release of solvents by Escherichia coli in an organic-aqueous two-liquid-phase system and substrate specificity of the AcrAB-TolC solvent-extruding pump, J. Bacteriol. 182 (2000) 4803-4810.

[70] Walker R.A., Lawson A.J., Lindsay E.A., Ward L.R., Wright P.A., Bolton F.J., Wareing D.R.A., Corkish J.D., Davies R.H., Threlfall E.J., Decreased susceptibility to ciprofloxacin in outbreak-associated multiresistant Salmonella typhimurium DT104, Vet. Rec. 147 (2000) 395396.

[71] White D.G., Goldman J.D., Demple B., Levy S.B., Role of the $a c r A B$ locus in organic solvent 
tolerance mediated by expression of marA, soxS, or robA in Escherichia coli, J. Bacteriol. 179 (1997) 6122-6126.

[72] Wiuff C., Madsen M., Baggesen D.L., Aarestrup F.M., Quinolone resistance among Salmonella enterica from cattle, broilers, and swine in Denmark, Microb. Drug Resist. 6 (2000) 11-17.

[73] Zgurskaya H.I., Nikaido H., Bypassing the periplasm: reconstitution of the AcrAB multidrug efflux pump of Escherichia coli, Proc. Natl. Acad. Sci. USA 96 (1999) 7190-7195.
[74] Zgurskaya H.I., Nikaido H., AcrA is a highly asymmetric protein capable of spanning the periplasm, J. Mol. Biol. 285 (1999) 409-420.

[75] Zgurskaya H.I., Nikaido H., Cross-linked complex between oligomeric periplasmic lipoprotein AcrA and the inner-membrane-associated multidrug efflux pump AcrB from Escherichia coli, J. Bacteriol. 182 (2000) 4264-4267.

[76] Zgurskaya H.I., Nikaido H., Multidrug resistance mechanisms: drug efflux across two membranes, Mol. Microbiol. 37 (2000) 219-225. 